\title{
Irregular Attractors
}

\author{
VADIM S. ANISHCHENKO* and GALINA I. STRELKOVA \\ Laboratory of Nonlinear Dynamics, Department of Physics, Saratov State University, \\ Astrakhanskaya str., 83, 410026 Saratov, Russia
}

(Received 20 September 1997)

\begin{abstract}
In this paper the definition of attractor of a dissipative dynamical system is introduced. The classification of the existing types of attractors and the analysis of their characteristics are presented. The discussed problems are illustrated by the results of numerical simulations using a number of real examples that provides the possibility to understand easily the main properties, similarities and differences of the considered types of attractors.
\end{abstract}

Keywords: Dynamical system, Attractor, Hyperbolic attractors, Lorenz type attractors, Quasiattractors, Strange nonchaotic attractors, Nonstrange chaotic attractors

\section{INTRODUCTION}

One of the main methods for investigation of selfoscillatory systems is the statement of equations describing their dynamics and analysis of their solutions. Therefore, the study of the field of mathematics named "Dynamical systems" is the basic part of fundamental training on the theory of nonlinear oscillations. In the classical theory of oscillations the study of periodic and quasiperiodic regimes which are important for the description of the phenomena of generation and modulation of oscillations was and remains the central problem. From this point of view, the mathematical images of these oscillatory regimes (a limit cycle and an $n$-dimensional torus) were not the main objects for investigation. They only provided an alternative representation of the above-mentioned regimes in the phase space of the systems under consideration. Really, one can define the main properties of periodic oscillations using a segment of one of the phase coordinates $x(t)$ on a finite time interval $0 \leq t \leq T$ ( $T$ - period of oscillations) together with the Fourier-spectrum or auto-correlation function of initial regime. In this sense quasiperiodic oscillations are only slightly different. For the observation time $T$ of the realization $x(t)$ it is necessary to choose the largest of the characteristic times that corresponds to the minimal basic frequency in the spectrum. In other words, the availability of a good oscillograph and spectrum analyzer allows investigators to get a complete information about the properties of generators including modulation effects.

* Corresponding author. 
When the dynamical chaos was discovered the situation dramatically changed (Schuster, 1984; Lichtenberg and Lieberman, 1983; Anishchenko, 1990; 1995). Chaotic oscillations are not periodic or quasiperiodic. Therefore, observation $x(t)$ during any finite time interval does not provide complete information. Moreover, it is very difficult to predict the specific observation times during which it is possible to determine the features of oscillatory regime. In this situation it is useful to analyze in detail the geometric image of a selfoscillatory regime in the system phase space, i.e., attractor. Note, that analyzing of the geometric structure of attractors being the images of selfoscillations in dissipative dynamical systems cannot provide full information about oscillations being, however, substantially more effective compared to the time series analysis.

As known, a so-called strange attractor (Lorenz, 1963; Ruelle and Takens, 1971) is associated with the image of dynamical chaos. Originally all nontrivial self-oscillatory regimes, whose general property is the absence of periodicity in time, were related with the image of the strange attractor. Later there came the understanding that chaotic self-oscillations may be substantially different in their properties. And it definitely leads to the difference in structure and properties of the corresponding attractors. So, for example, it has become clear that strange attractor is the image of some "ideal" chaos satisfying a number of rigorous mathematical requirements. It has been established that in real systems the regime of strange attractor in the strict sense of mathematical definition cannot be realized. What we observe in experiments is more often the regimes of a so-called quasihyperbolic attractor or quasiattractor, which are more complicated and cannot be rigorously described in terms of mathematics (Afraimovich and Shil'nikov, 1983; Afraimovich, 1989; 1990). A distinctive feature of strange, quasihyperbolic and quasiattractors is exponential instability of phase trajectories and the fractal dimension. Exponential instability is a criterium of chaotic behavior of the system in time. The fractal metric dimension shows that the attractor is a complex geometric object which is not a manifold. Since our knowledge of deterministic chaos is related just with these properties, one does not pay a significant attention to the differences between geometric characteristics of the attractor and temporal characteristics of the system's dynamics. Nevertheless, recently the attention of researchers has been attracted by the fact that non-periodic oscillations can possess asymptotic stability in the presence of complex geometry of the attractor and, on the contrary, they can be exponentially unstable and correspond to the attractor which is a simple geometric object (a manifold) (see, Farmer et al., 1983; Grebogi et al., 1984).

It is appropriate to introduce a definition of "strangeness" of the attractor in terms of its geometric structure and without connection with the system's dynamics. In the paper by Grebogi et al. (1984) such a definition is formulated: "Strange attractor is an attractor which is not a finite set of points and not piecewise differentiable. We say that an attractor is piecewise differentiable if it is a piecewise differentiable curve or surface, or a volume bounded by a piecewise differentiable closed surface".

Taking into account the importance of the analysis of complex non-periodic regimes of oscillations in dynamical systems it is very useful to classify in detail the types of attractors and formulate their definitions and basic properties.

In this paper we present definitions, properties and examples of non-trivial attractors of different types which are realized in differential and discrete dissipative nonlinear dynamical systems with finite number of degrees of freedom.

The paper is organized as follows. We start with a definition of a dynamical system attractor in Section 1. Regular attractors are discussed in Section 2. The main part of the work is Section 3 that is devoted to the analysis of strange chaotic attractors. In this section we describe robust hyperbolic strange attractors, quasi-hyperbolic attractors (Lorenz type attractors) and quasiattractors. In Section 4 chaotic nonstrange and 
strange nonchaotic attractors are analyzed. And finally, in Section 5 we formulate conclusions.

The results presented in this paper have the aim to provide the necessary information available for researchers being specialists in the experiments with nonlinear dynamical systems.

\section{WHAT IS AN ATTRACTOR?}

The time evolution of the state of a system with $N / 2$ degrees of freedom is described by either a deterministic system of differential equations or $N$-dimensional maps:

$$
\frac{\mathrm{d} x_{i}}{\mathrm{~d} t}=\dot{x}_{i}=f_{i}\left(x_{1}, \ldots, x_{N}, \mu_{1}, \ldots, \mu_{k}\right),
$$

or

$$
\begin{aligned}
x_{n+1}^{i} & =f_{i}\left(x_{n}^{1}, x_{n}^{2}, \ldots, x_{n}^{N}, \mu_{1}, \ldots, \mu_{k}\right), \\
& i=1,2, \ldots, N .
\end{aligned}
$$

Here, $x_{i}(t)$ (or $x_{n}^{i}$ ) are variables uniquely describing the system's state (its phase coordinates), $\mu_{l}$ are system parameters, $f_{i}(x, \mu)$ are smooth and, in general case, nonlinear functions. A solution of the system (1) exists, it is unique for the given initial conditions $x_{i}(0)$ (or $x_{0}^{i}$ ) and smoothly depends on the initial conditions (Cauchy theorem).

Time evolution of the system can be uniquely related with the phase trajectory in $N$-dimensional Cartesian space $\mathfrak{R}^{N}$, whose coordinates are phase variables. The trajectory starts from the given initial condition $x_{i}(0)$ (or $\left.x_{0}^{i}\right), i=1,2, \ldots, N$.

We will consider only self-oscillatory regimes of the system motion. From the physical point of view, the latter means that in the system there exist some steady-state oscillations whose characteristics do not depend, to a certain extent, on the choice of initial state. We shall also consider the regime of a stable equilibrium state being a limit case of selfoscillatory regime. As we will see, the notion selfoscillatory regime introduced by Andronov (Andronov et al., 1981) is the classical physical interpretation of the definition of a dynamical system attractor.

Let us examine the phase space $\mathfrak{R}^{N}$ of system (1). All the values of system's parameters $m_{k}$ are fixed. Let $G_{1}$ be some finite (or infinite) region belonging to $\Re^{N}$ and including a subregion $G_{0}$. The regions $G_{1}$ and $G_{0}$ satisfy the following conditions: 1 . For any initial conditions $x_{i}(0)$ (or $x_{0}^{i}$ ) from the region $G_{1}$ all phase trajectories will reach sooner or later (in theory as $t \rightarrow \infty($ or $n \rightarrow \infty)$ ) the region $G_{0}$. 2. If a phase trajectory belongs to the region $G_{0}$ at the moment $t=t_{1}\left(n=n_{1}\right)$, then it will always belong to $G_{0}$, i.e., for any $t \geq t_{1}$ (or $n \geq n_{1}$ ) the phase trajectory will be in the region $G_{0}$ (Afraimovich, 1989; 1990).

If the conditions 1 and 2 are satisfied, then the region $G_{0}$ is called an attractor of a dynamical system (1). In other words, the attractor $G_{0}$ is the invariant with respect to the law (1) bounded set of system's trajectories, which any trajectories from $G_{1}$ approach and remain in. The region $G_{1}$ is called the region (or basin) of attraction for the attractor $G_{0}$. According to the definition, only transient nonstationary types of motions can exist in the region $G_{1}$. The region $G_{0}$ corresponds to the steadystate (limit) types of motions. In this sense one can say that the attractor $G_{0}$ is the isolated limit set of the phase trajectories of system (1). Any types of the system motion in the vicinity of the attractor have only a transient character and as a result, the phase trajectories are attracted by the region $G_{0}$ as $t \rightarrow \infty(n \rightarrow \infty)$. Hence, the name appears "attractor".

The given definition of the attractor requires some comments. Let us examine a stable ergodic two-dimensional torus as an example of attractor. Any point on the ergodic torus surface belongs to the attractor. By varying the system's parameter, turn to the regime of the resonance structure on the torus (let it be the resonance $1: 1$ ). In the Poincare section the resonance $1: 1$ corresponds to the closure of unstable separatrixes of a saddle on a stable node. The problem is whether these separatrixes belong to the attractor or the attractor is a stable point being an image of the resonant limit 
cycle. From the viewpoint of rigorous mathematics, the first is correct! But in experiments we will observe only the stable limit cycle that is connected with the attractor in our understanding (Andronov et al., 1981; Arnold et al., 1986).

\section{REGULAR ATTRACTORS}

Before deterministic chaos was discovered only three types of stable steady-state solutions of the dynamical system (1) were known: an equilibrium state when after a transient process the system reaches a stationary (non-changing in time) state; a stable periodic solution and a stable quasiperiodic solution. In these cases the corresponding attractors are a point in the phase space, a limit cycle and a limit $n$-dimensional torus. The Lyapunov characteristic exponents (LCE) signature of a phase trajectory will be as follows (Anishchenko, 1990; 1995):

$$
\begin{aligned}
& \text { " } \\
& \text { - for an equilibrium state, }
\end{aligned}
$$

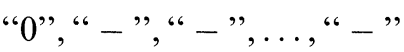

$$
\begin{aligned}
& \text { - for a limit cycle, } \\
& \underbrace{\text { “0”, “0”, ., "0" }, " ~}_{n}, ", \ldots, ", \quad \text { - } \\
& \text { - for an } n \text {-dimensional torus, } n \geq 2 \text {. }
\end{aligned}
$$

As we will see further, strange chaotic attractors of a complex geometrical structure correspond to non-periodic solutions of the system (1). They have at least one positive Lyapunov exponent and, as a consequence, a fractional dimension that can be evaluated by using Kaplan-Yorke's definition (Kaplan and Yorke, 1979):

$$
D=j+\frac{\sum_{i=1}^{j} \lambda_{i}}{\left|\lambda_{j+1}\right|},
$$

where $j$ is the largest integer number for which the sum $\lambda_{1}+\lambda_{2}+\cdots+\lambda_{j} \geq 0$. The dimension $D$ calculated from formula (2) is one of the fractal dimensions of the set and is called Lyapunov's dimension. In general case, it is a lower bound for the metric dimension of the attractor. If we apply formula (2) to the three types of the attractors indicated above, we will then have the dimension that is equal to zero for the attractor being a point, $D=1$ for the limit cycle attractor and $D=n$ for the $n$-dimensional torus. It is very interesting to note that in all cases the fractal dimension is strictly equal to the metric dimension of the attractors. The indicated types of the solutions are asymptotically stable. The dimension $D$ of the corresponding attractors is defined by an integer number and strongly coincides with the metric dimension. All these facts allow us to say that the attractors indicated above are regular. If one of the formulated conditions is violated, then the attractor is excluded from the group of regular attractors. As it has become clear now non-regular (strange) attractors require special classification (Afraimovich, 1984; 1989; 1990; Shil'nikov, 1993).

\section{STRANGE CHAOTIC ATTRACTORS}

A new type of attractor of the dynamical system (1) was first revealed by Lorenz in 1963 when he was investigating numerically the Lorenz model (Lorenz, 1963). A rigorous proof of the existence of non-periodic solutions of system (1) was given by Ruelle and Takens in 1971. They also introduced the notion of strange attractor as the image of deterministic chaos (Ruelle and Takens, 1971). Since that time, very often the phenomenon of deterministic chaos and the concept of strange attractor are definitely connected to each other. However, this is not always correct and needs some explanations.

\subsection{Robust Hyperbolic Attractors}

If we read the work (Ruelle and Takens, 1971) very carefully, we will realize that a proof of the strange attractor existence was given under the strong suggestion that the dynamical system (1) was 
robust hyperbolic. What does it mean? The system is hyperbolic if all of its phase trajectories are saddle. A point as an image of a trajectory in the Poincare section is always a saddle. Robustness means that when the right-hand parts in (1) are slightly perturbed or control parameters are slightly varied, all the trajectories remain saddle.

Speaking more strictly, hyberbolic attractors should satisfy the following three conditions (Afraimovich, 1989; 1990):

1. A hyperbolic attractor consists of a continuum of "unstable leaves", or curves, which are dense in the attractor and along which close trajectories exponentially diverge.

2. A hyperbolic attractor (in the neighborhood of each point) has the same geometry defined as a product of the Cantor set on an interval.

3. A hyperbolic attractor has a neighborhood foliated into "stable leaves" along which the close trajectories converge to the attractor.

Robustness means that properties $1-3$ hold under perturbations.

Figure 1 represents a saddle trajectory $\Gamma$ and the corresponding points $Q_{i}$ of its intersection with the secant Poincare surface $S$ and also illustrates the local behavior of stable and unstable manifolds of a saddle point $Q_{i}$. But the condition that the point $Q_{i}$ of intersection of $\Gamma$ with $S$ is locally a robust saddle is not enough for robust hyperbolicity! Certain conditions upon global (non-local) properties of stable and unstable manifolds are needed. Let us consider Fig. 2. Due to the presence of attractor, stable and unstable manifolds $W_{\mathrm{s}}$ and $W_{\mathrm{u}}$ are to be concentrated in the region of the attractor $G_{0}$. At the same time they can intersect with the appearance of homoclinic points (surfaces) by forming so-called homoclinic structures. These structures in robust hyperbolic systems must be robust. This means that from the topological viewpoint, the intersection structure of $W_{\mathrm{s}}$ and $W_{\mathrm{u}}$ must correspond to Fig. 2(a) and should not change qualitatively under perturbations! The cases in Figs. 2(b) and (c) are excluded as they characterize two non-robust phenomena, namely, the phenomenon of closure of the manifolds with the loop formation (Fig. 2(b)) and the phenomenon of tangency of the stable and unstable manifolds (Fig. 2(c)).

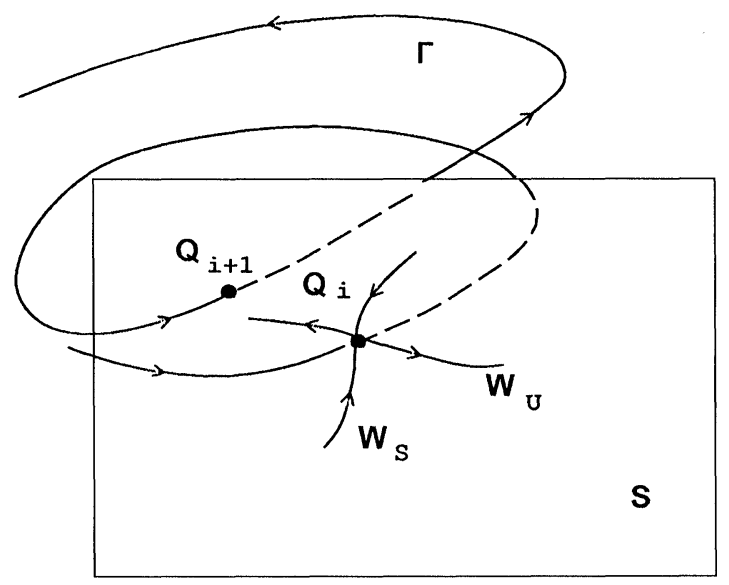

FIGURE 1 A saddle point $Q_{i}$ as the image of a hyperbolic trajectory in the Poincare section.

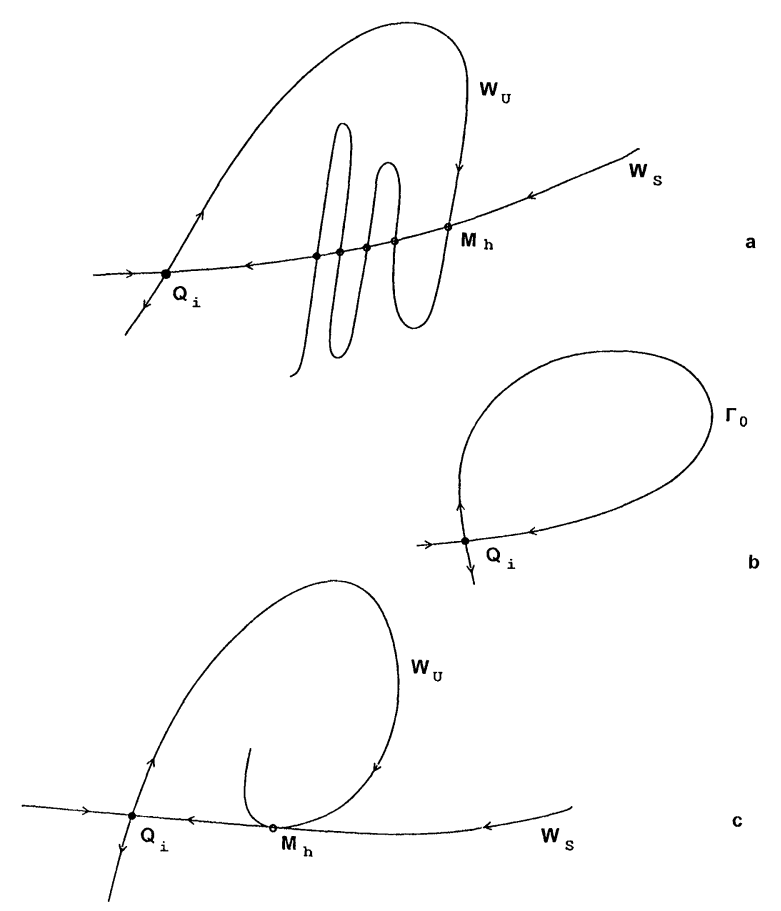

FIGURE 2 Three possible cases of the intersection of the stable and unstable separatrixes of the saddle point $Q_{i}$ in the Poincare section. 
If non-local properties of the manifolds lead to the non-robust situations shown in Figs. 2(b) and (c) when the dynamical system is perturbed, bifurcations of system's solutions are possible (Gavrilov and Shil'nikov, 1972; 1973). In robust hyperbolic systems no bifurcations should occur. If small perturbations are introduced, the trajectory $\Gamma$ always remains saddle, the latter corresponding to the case shown in Fig. 2(a). As we will see later, the non-robust cases (Figs. 2(b) and (c)) cause the appearance of more complicated chaotic attracting sets, i.e., quasiattractors (Afraimovich, 1984; 1989; 1990).

Therefore, it is necessary to understand that strange (according to Ruelle-Takens) attractors are always robust hyperbolic limit sets. The main feature in which strange chaotic attractors differ from regular ones is exponential instability of the phase trajectory on the attractor. In this case the LCE spectrum includes at least one positive exponent:

$$
\begin{gathered}
\text { " }+, " 0 ", ", ", \ldots, ",-", \\
D=2+\lambda^{+} /\left|\lambda_{3}\right|>2 .
\end{gathered}
$$

As seen from Kaplan-Yorke's formula, fractal dimension of an attractor will always be more than 2 and, in general case, will not be defined by an integer number. A minimal dimension of the phase space in which a strange attractor can be "embedded" equals 3. Therefore, the regime of deterministic chaos can be observed in differential dynamical systems which have the dimensionality $N \geq 3$.

In mathematics at least two examples of robust hyberbolic attractors are known. These are Smale-Williams attractor (Smale, 1967) and Plykin attractor (Plykin, 1980). Unfortunately, up to now in real systems the regime of rigorously hyperbolic robust chaos has not been revealed! "Truly" strange attractors are an ideal but still unattainable model of deterministic chaos. In real life, as usual, everything is more complicated compared with idealization.

\subsection{Quasihyperbolic Attractors. Lorenz Type Attractors}

There is a finite number of dynamical systems that have almost hyperbolic attractors (quasihyperbolic attractors). Such attractors do not contain stable regular trajectories (points, cycles etc.) and are closest in their structure and properties to robust hyperbolic attractors. As examples, we can indicate here the Lorenz attractor (Lorenz, 1963; Shil'nikov, 1980), Belykh and Lozi attractors (Lozi, 1978; Belykh, 1982; 1995). For quasihyperbolic attractors at least one of three conditions of hyperbolicity is violated. In particular, for the Lorenz attractor the second condition is not valid. However, Lorenz type attractors were revealed in a number of systems and from the experimental point of view, we can treat them as the examples of "truly" strange attractors (Shil'nikov, 1980; Williams, 1977; Cook and Roberts, 1970).

It is surprising but it is a fact that it is the chaotic attractor in the Lorenz model that is closest in its properties and structure to robust hyperbolic attractors. In the Lorenz attractor all trajectories are saddle and when one varies parameters, no stable points or cycles are born (Bykov and Shil'nikov, 1989; Afraimovich, 1984; 1989; 1990).

The Lorenz equations were first obtained from the Navier-Stokes's equations while solving the problem of thermal convection and have the following form:

$\dot{x}=-\sigma(x-y), \quad \dot{y}=r x-y-x z, \quad \dot{z}=x y-b z$,

where $\sigma, b$ and $r$ are control parameters. Some laser models as well as the model of disc dynamo can be reduced to the equations of the type (3) (Shil'nikov, 1980; Cook and Roberts, 1970).

System (3) is invariant with respect to the transformation $(-x,-y, z) \rightarrow(x, y, z)$ and is characterized by three equilibrium states. Let us fix the values of the parameters as $\sigma=10, b=8 / 3$. While studying the behavior of system's trajectories when the parameter $r$ is varied, over the critical point 
$r_{\mathrm{cr}} \cong 24,74$ the only chaotic attractor, i.e., the Lorenz attractor, is realized. The basin of its attraction is the entire phase space. The Lorenz attractor is the attracting set consisting of the phase trajectories which are characterized by the individual exponential instability. The properties indicated above do not change when one varies the parameters in a finite range of their values and do not depend on initial conditions. Figure 3 shows a projection of the Lorenz attractor on the plane $(x, z)$ and the basin of its attraction.

The Lorenz attractor demonstrates practically all properties and qualities of robust hyperbolic attractors:

1. The presence of a denumerable set of separatrix loops of equilibrium states of the Lorenz system does not lead to the birth of stable regular attractors when any perturbations are added.

2. When the parameters of the system (3) are varied in a finite range of their values, no bifurcations occur in the Lorenz attractor and no other stable attracting subsets appear.

3. When the equations are slightly perturbed or external noise of small intensity is added, the

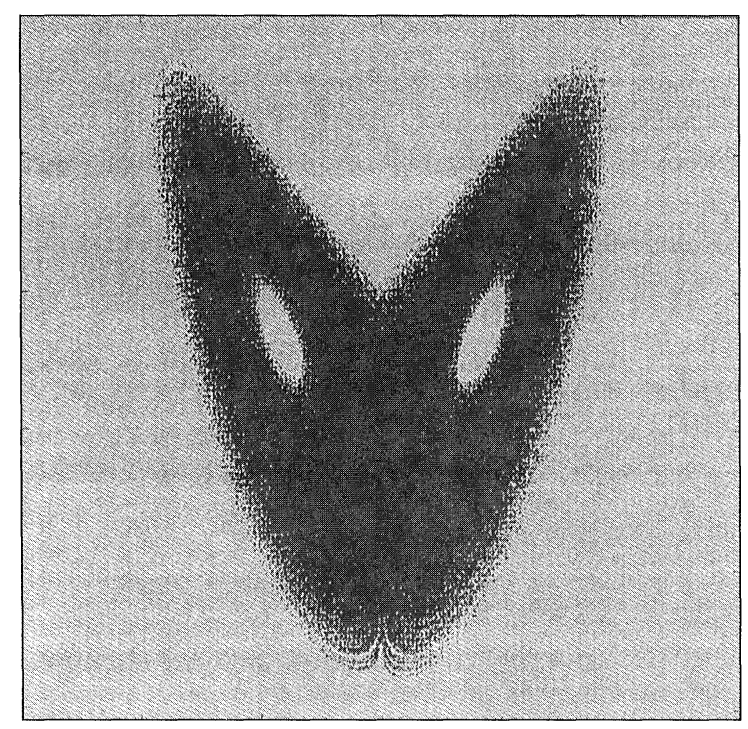

FIGURE 3 Projection of the Lorenz attractor on the plane $(x, z)$ (black color) and the basin of its attraction (grey color) in the Poincare section $y=0$ for $r=28, \sigma=10, b=8 / 3$. changes in the attractor's structure are also small. The own dynamical nature of the chaotic behavior is much stronger than that of the nondynamical chaos added from outside.

4. For the Lorenz attractor it is possible to construct a smooth function of distribution density $p(x, y, z)$, i.e., the probability measure of the attractor. Small perturbations of the flow (3) cause small changes in the probability measure according to the theoretical results which were formulated for hyperbolic systems by Kifer (1974).

The Lorenz attractor has the classical spectrum of the Lyapunov characteristic exponents (LCE):

$$
\begin{aligned}
& \lambda_{1}=0.9, \quad \lambda_{2}=0, \\
& \lambda_{3}=-14.57 \quad(r=26) .
\end{aligned}
$$

It gives Lyapunov's dimension $D=2.06$. The fact that the fractal part of dimension is close to 0 is explained by the strong contraction of the flow in dissipative system (3)

$$
\operatorname{div} \mathbf{F}=-(\sigma+b+1) .
$$

This circumstance explains the fact that the Poincare map of the Lorenz attractor is very close to a one-dimensional map. Due to the fact that $\operatorname{div} \mathbf{F}($ see (5)) does not depend on phase variables, the birth of the regime of two-frequency quasiperiodic oscillations in system (3) is impossible. Therefore, the set of non-singular phase trajectories of the system includes only points, cycles and the Lorenz attractor.

Note that from experimental viewpoint, system (3) displays practically robust hyperbolic chaos in a finite range of values of its control parameters. The bifurcation diagram of system (3) is shown in Fig. 4 (Bykov and Shil'nikov, 1989). The shaded region in the parametric space corresponds to the existence of Lorenz attractor, while outside of this region the properties of the chaotic attractor will be essentially different. In particular, bifurcation line $l_{3}$ in Fig. 4 corresponds to the transition "Lorenz attractor - quasiattractor". 
Let us illustrate the typical characteristics and properties of Lorenz attractor. The LCE spectrum does not change under variation of initial conditions because the Lorenz attractor is the only one and the basin of its attraction is the entire phase space (see Figs. 4 and 5).

The LCE spectrum does not practically change as one varies the system's control parameters in the region of Lorenz attractor existence (see Fig. 4). These properties visually illustrate the robustness of Lorenz attractor in experiments:

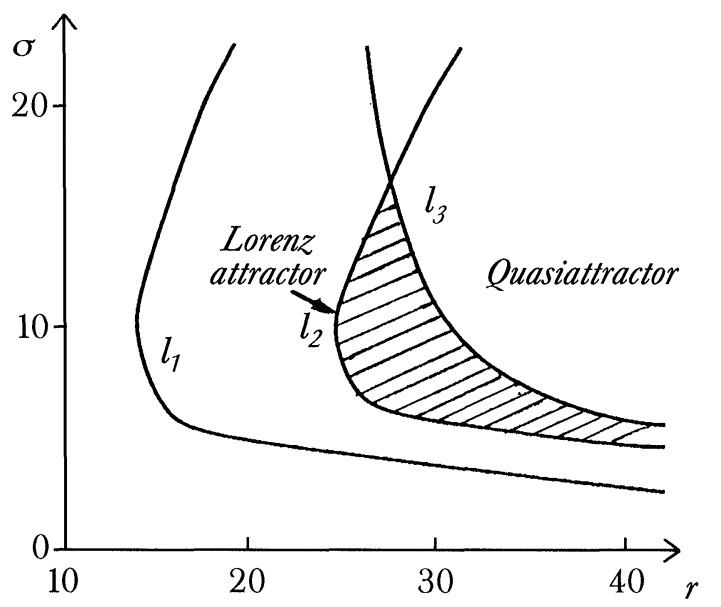

FIGURE 4 Bifurcation diagram of the Lorenz system on the plane of parameters $z, \sigma$ for $b=8 / 3 . l_{1}$ is the line of the existence of a symmetrical separatrix loop of zero equilibrium state; $l_{2}$ is the line of the birth of the Lorenz attractor; $l_{3}$ is the line of the bifurcation transition to quasiattractor.

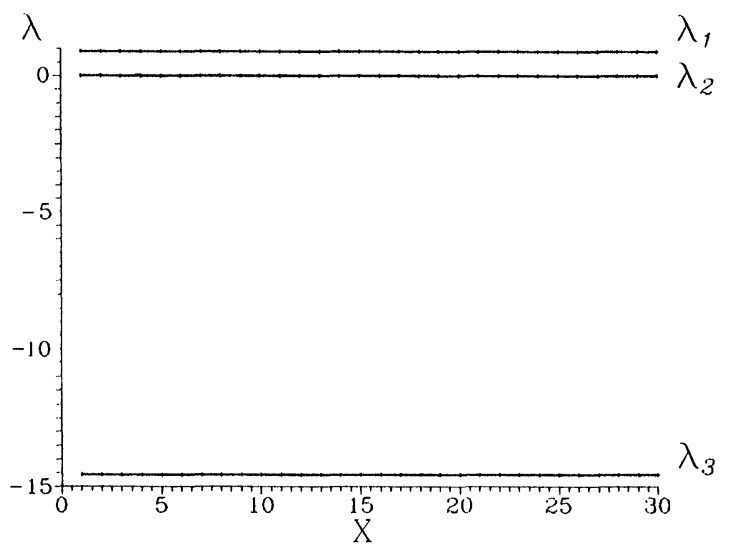

FIGURE 5 Dependence of the LCE spectrum of the Lorenz attractor on the values of $x$ coordinate for $r=28, \sigma=10$, $b=8 / 3$. general properties of the attractor hold under variation of the parameters and initial conditions; no bifurcations of the attractor occur.

Autocorrelation function and power spectrum of Lorenz attractor presented in Fig. 6 are typical for intermixing systems. The autocorrelation function almost exponentially decreases without oscillations with the increase of time (see Fig. 6(a)). The power spectrum is a continuous decreasing function of frequency and does not contain pronounced peaks at any characteristic frequencies (see Fig. 6(b)).

All the characteristics and properties of the Lorenz attractor do not practically change in the presence of additive (or multiplicative) noise with small intensity. Figure 7 represents the plots for the stationary two-dimensional probability density $p(x, z)$ of Lorenz attractor in the absence and in the presence of additive noise introduced into the three equations of system (3) (Anishchenko, 1995).

\subsection{Quasiattractors}

The so-called quasiattractors (Afraimovich and Shil'nikov 1983; Shil'nikov, 1993) are most typical in experiments. They illustrate experimentally observed chaos in the majority of dynamical systems (Schuster, 1984; Lichtenberg and Lieberman, 1983; Anishchenko, 1990; 1995; Neimark and Landa, 1989; Rabinovich and Trubetskov, 1984). In the systems with quasiattractors the regimes of deterministic chaos, which are characterized by the exponential instability of trajectories and a fractal geometry of the attractor, are realized. From this point of view, the characteristics of the indicated regimes of self-oscillations are identical to the general ones of robust hyperbolic attractors and Lorenz type attractors. However, there are very essential and principal differences which it is necessary to take into consideration to avoid incorrect explanations of experimental results. A feature of quasiattractors is the co-existence of a denumerable set of different chaotic and regular attractors in a bounded element of the system phase space volume when the system's parameters are fixed. This set of all co-existing limit subsets of 

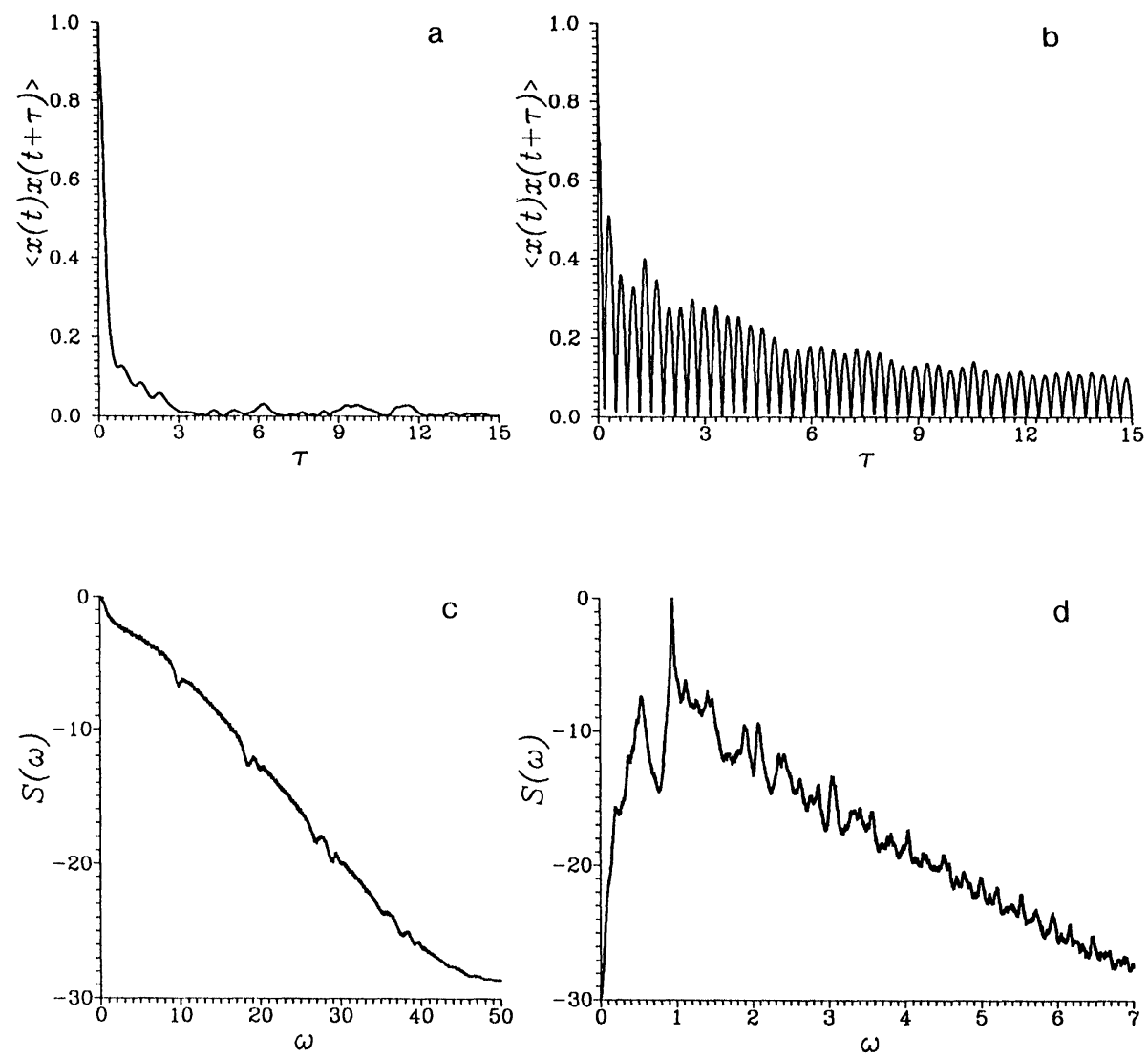

FIGURE 6 Autocorrelation function and power spectrum for the Lorenz attractor $(a, c)$ and for Anishchenko-Astakhov's oscillator $(b, d)$.

trajectories in the bounded region $G_{0}$ of phase space, which all or almost all the trajectories from the region $G_{1}$ including $G_{0}$ approach, is called the quasiattractor of the dynamical system.

Quasiattractors are characterized by a very complex structure of embedded basins of attraction. But the complexity is wider than this fact. Under variation of system's parameters in a finite range of their values the cascades of different bifurcations of both regular and chaotic attractors are realized. Accordingly, the bifurcational reorganization of their basins of attraction takes place. The reason for such a complexity of quasiattractors is the effects of homoclinic tangency of stable and unstable manifolds of saddle points in the Poincare section which take place on the set of parameter values of non-zero measure
(Gavrilov and Shil'nikov, 1972; 1973; Afraimovich, 1984; 1989; 1990).

If one takes into account that the basins of attraction of co-existing limit sets can have fractal boundaries and occupy very narrow regions in phase space, then it becomes clear how important the role of accuracy in numerical experiments and the influence of external noises is. Let us demonstrate the properties of quasiattractors using a series of examples.

Let us explore a typical system whose chaotic dynamics fully illustrates Shilnikov's theorem about the properties of dynamical systems with a saddle-focus separatrix loop of the equilibrium state (Anishchenko, 1990; 1995). This system is called a modified oscillator with intertial nonlinearity (Anishchenko-Astakhov's oscillator) and is 

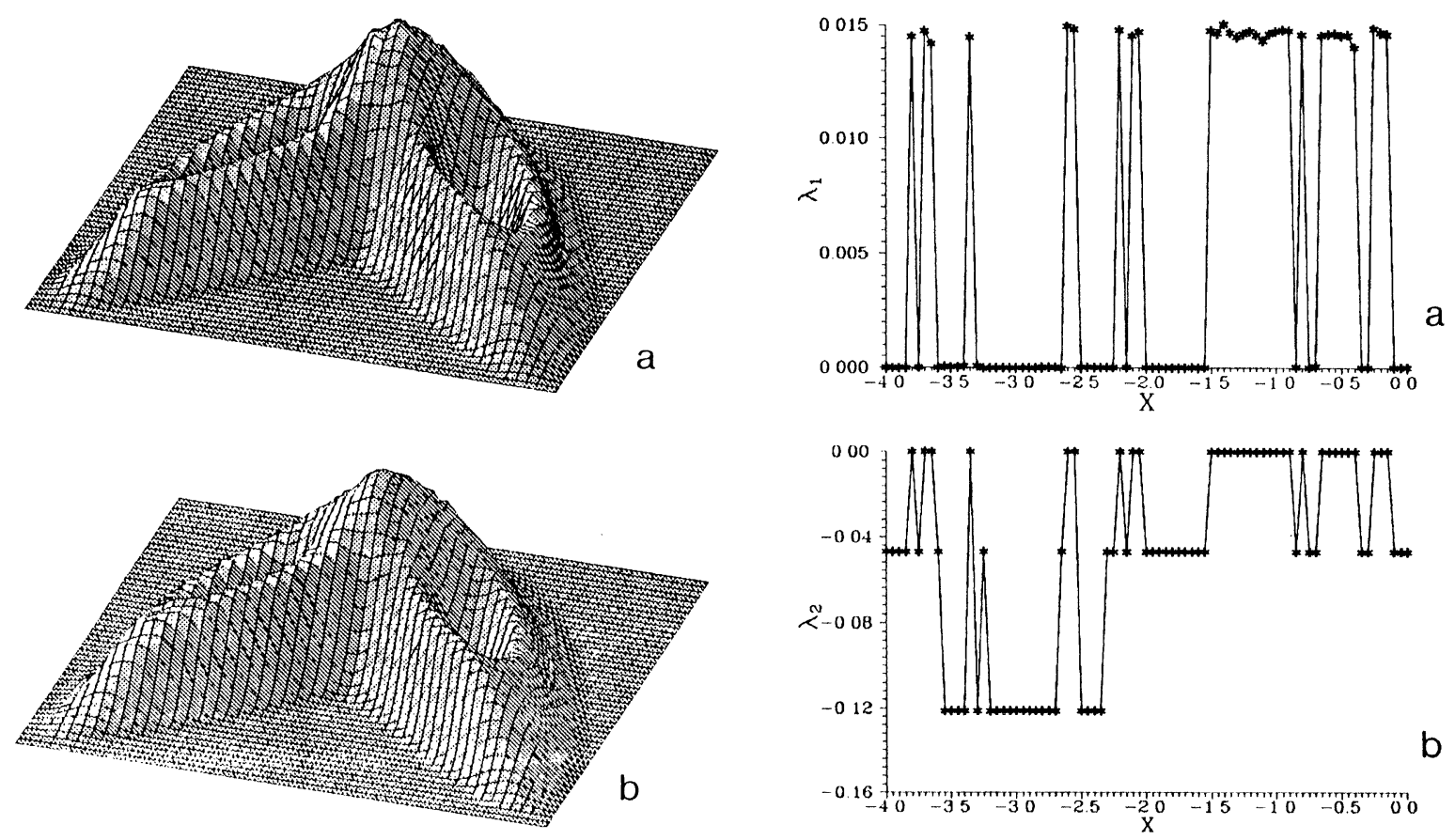

FIGURE 7 Stationary two-dimensional probability density $p(x, z)$ of the Lorenz attractor in the absence of noise (a) and in the presence of additive white noise with intensity $d=0.8$ (b).

a three-dimensional two-parametric differential system described by the following equations:

$$
\begin{gathered}
\dot{x}=m x+y-x z, \quad \dot{y}=-x, \\
\dot{z}=-g z+g I(x) x^{2},
\end{gathered}
$$

where

$$
I(x)= \begin{cases}1, & x>0 \\ 0, & x \leq 0 .\end{cases}
$$

Let us fix the parameters as $m=1.42, g=0.097$ and calculate the exponents of the LCE spectrum as a function of initial conditions. The results are shown in Fig. 8 and represent the co-existence of chaotic and periodic oscillatory regimes. The more detailed analysis of the results in Fig. 8 shows that for $-2.0<x<0$ we can observe the regime of one of the limit cycles and the chaotic regime, while for $-4.0<x<-2.0$ a limit cycle of another family is added (compare the values of the third exponent of the LCE spectrum). More visually this situation

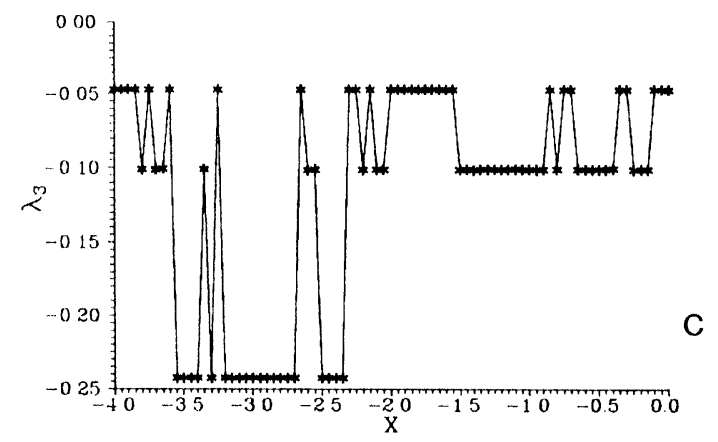

FIGURE 8 The LCE spectrum of system (6) as a function of $x$ coordinate for the parameters $m=1.42, g=0.097$.

can be illustrated by Fig. 9. This figure represents projections of the three co-existing attractors in system (6) and basins of their attraction. Indeed, period 1 and period 2 limit cycles and the chaotic attractor co-exist in the system.

Due to nonrobustness of system (6) the all of its limit subsets undergo bifurcations as the parameters are varied. To illustrate this fact we present the dependence of the LCE spectrum exponents on the parameter $m$ shown in Fig. 10.

The fact that the exponent $\lambda_{1}$ is equal to 0 testifies to the birth of one of the sets of limit cycles 
which demonstrate the cascades of period doubling bifurcations. With this, $\lambda_{1}$ equals to 0 , while $\lambda_{2}$ takes different negative values. Bifurcations of the attractors are accompanied by the changes in the structure of their basins of attraction whose boundaries become fractal.

The presence of stable and saddle cycles in a quasiattractor together with chaotic limit subsets manifests itself in the structures of autocorrelation function $(\mathrm{ACF})$ and power spectrum. The results

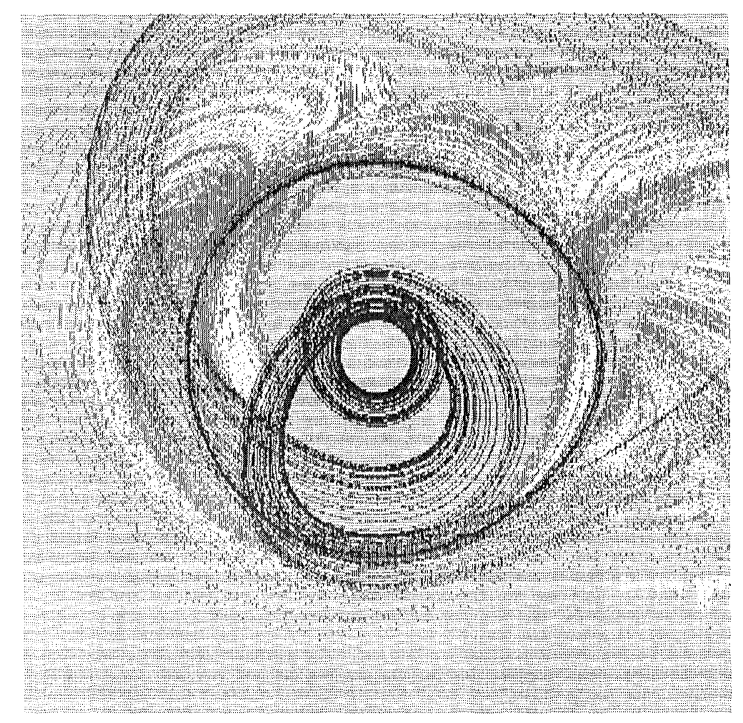

FIGURE 9 Projections of the co-existing attractors in system (6) for $m=1.42, g=0.097$ and the structure of the basins of their attraction in the Poincare section $z=1$.
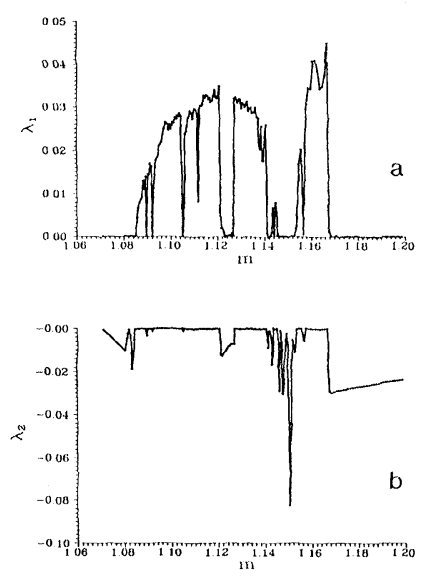

FIGURE 10 Lyapunov exponents $\lambda_{1}$ and $\lambda_{2}$ as a function of parameter $m(g=0.2)$ for system (6). calculated for the chaotic regime of system (6) at $m=1.5, g=0.2$ are presented in Figs. 6(b) and (d). The ACF decreases exponentially in average with time and the power spectrum is continuous. However, under a more careful consideration we can notice a periodic component in the ACF and sudden peaks at certain characteristic frequencies in the spectrum. Quasiattractor differs from Lorenz attractor by these peculiarities of ACF and power spectrum of chaotic regime, the latter being typical (compare the results presented in Fig. 6).

Fractality and riddling of the basin boundaries of the set of co-existing regular and chaotic attractors of the system cause a high sensitivity to noise perturbations. Let us consider the regime of the chaotic attractor in system (6) where $m=1.5$, $g=0.2$. Figure 11 represents the plots of the twodimensional probability density $p(x, y)$ in the absence of noise and in the case when Gaussian noise is introduced additively to the right-hand
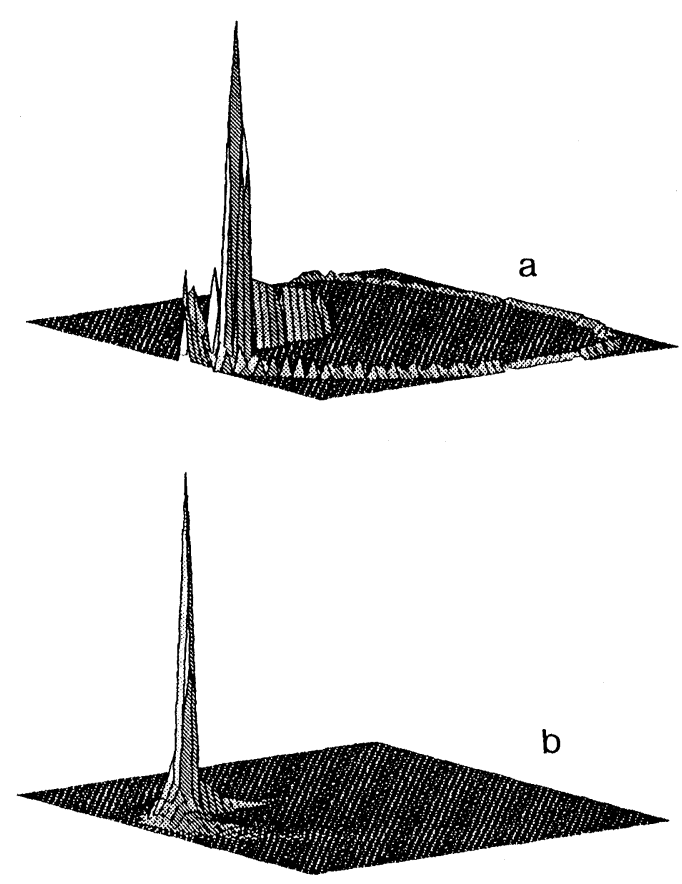

FIGURE 11 Probability distribution density $p(x, y)$ for the chaotic regime in Anishchenko-Astakhov's oscillator ( $m=1.5, g=0.2)$ in the absence of noise (a) and in the presence of additive noise with intensity $d=10^{-3}$ introduced to all the system (6) equations (b). 
parts of the three system equations. As seen from the figures, the introduction of the noise of small intensity leads to explicit changes in the structure of probability function.

We have chosen the regime where the finite number of attractors co-exist as a visual illustration of complexity of a quasiattractor. Theoretically a quasiattractor includes an infinite number of coexisting limit regimes which undergo an infinite sequence of different bifurcations when parameters are varied slightly. Also there can be the ranges of parameter values where (from the experimental point of view) the system has only one chaotic attractor that attracts all trajectories in the phase space. If we are able to observe such a regime in experiments and determine the region of parameters where it exists, we can then speak about the regime that is close to robust hyperbolic attractor.

As an example, examine a discrete dynamical system in the form of two coupled logistic maps (Strelkova and Anishchenko, 1997):

$$
\begin{aligned}
& x_{n+1}=1-\alpha x_{n}^{2}+\gamma\left(y_{n}-x_{n}\right), \\
& y_{n+1}=1-\alpha y_{n}^{2}+\gamma\left(x_{n}-y_{n}\right) .
\end{aligned}
$$

The only regime of hyperchaos can be realized in system (7) whose basin of attraction is a bounded rhombus on the parameter plane $\left(x_{n}, y_{n}\right)$. However, if we change the control parameters, the number of co-existing attractors increases abruptly and the structure of basins of their attraction becomes more complicated. The results are shown in Fig. 12. Figure 12(b), in particular, visually illustrates the influence of uncertainty in the choice of initial conditions on the system's behavior. For this purpose choose a small box denoted by 2 in Fig. 12(b) as a region of uncertainty in initial conditions from the basin of attraction of the quasiattractor and examine its evolution in time. We will obtain a combination of all three attractors of the system as the stationary regime! In experiments, in the presence of noise one of the three coexisting regimes will dominate randomly in the system's dynamics. This means that by choosing initial conditions with a finite accuracy the general property of dynamical chaos, i.e. the reproduc-
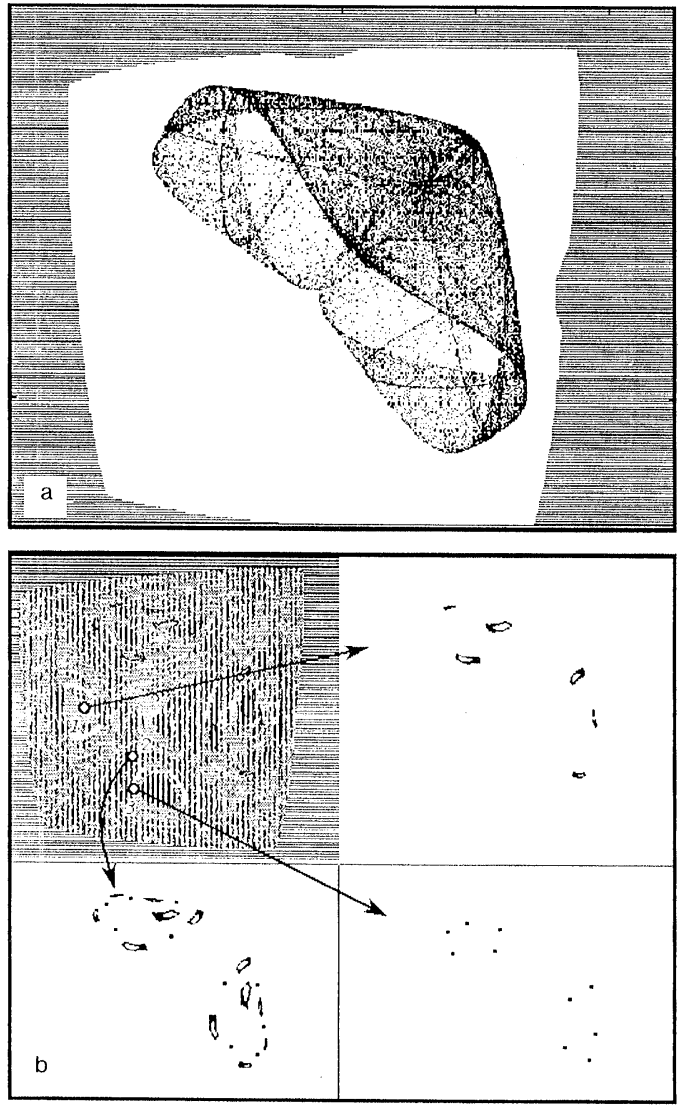

FIGURE 12 Hyperchaos in system (7) for parameters $\alpha=0.9, \gamma=0.285$ (a), the regime of the co-existence of attractors and the structure of the basins of their attraction in system (7) as $\alpha=0.78, g=0.2876$ (b) in phase space $\left(x_{n}, y_{n}\right)$. The numbers $1,2,3$ denote the regions of uncertainty in initial conditions leading to the corresponding limit sets indicated by arrows.

ibility from initial conditions, will be violated in such systems.

\section{STRANGE NONCHAOTIC AND CHAOTIC NONSTRANGE ATTRACTORS}

Chaotic attractors of the three types described above have two common principal properties. The first one is the complex geometric structure of an attractor (and, as a consequence, fractality of its metric dimension). The second property is the exponential instability of individual trajectories on 
the attractor. It is these properties that are used by researchers as a criterium for diagnostics of the regimes of deterministic chaos.

However, nonregular attractors as the mathematical images of complex dynamics are not restricted by the chaotic attractors described above. It has become clear that chaotic behavior in the sense of intermixing and the geometric "strangeness" of an attractor cannot be related with each other. Strange attractors in terms of their geometry can be nonchaotic due to the absence of exponential instability of phase trajectories. On the other hand, there are examples of intermixing dissipative systems whose attractors are not strange in a strict sense, that is, they are not characterized by the fractal structure and the fractal metric dimension.

In other words, there are examples of concrete dissipative dynamical systems whose attractors are characterized by the following properties:

1. An attractor has a regular geometric structure from the viewpoint of integer metric dimension. In addition, individual phase trajectories on the attractor are exponentially unstable in average.

2. An attractor is characterized by a complicated geometric structure. Here, trajectories on it are asymptotically stable. There is no intermixing.

The first type is called a chaotic nonstrange attractor $(C N A)$. The second one is called a strange nonchaotic attractor (SNA).

\subsection{Chaotic Nonstrange Attractors}

Chaotic attractors which are not strange from the viewpoint of their geometry have been known for a long time (Farmer et al., 1983; Grebogi et al., 1984; 1985), but by now they nave been studied insufficiently. The modified Arnold's map (Farmer et al., 1983 ) is an example of a dynamical system with CNA. This map is a well-known "cat map" with a nonlinear periodic term:

$$
\begin{aligned}
& x_{n+1}=x_{n}+y_{n}+\delta \cos 2 \pi y_{n}, \quad \bmod 1, \\
& y_{n+1}=x_{n}+2 y_{n}, \quad \bmod 1 .
\end{aligned}
$$

If $\delta<1 / 2 \pi$, then map (8) is a diffeomorphism on a torus. In other words, map (8) is one-to-one (reversible) and transforms a unit square on the plane $\left(x_{n}, y_{n}\right)$ into itself. Map (8) is dissipative, that is an area element contracts with each iteration. This property is proved easily if one calculates the Jacobian:

$$
J=\left|\begin{array}{cc}
1 & 1-2 \pi \delta \sin 2 \pi y_{n} \\
1 & 2
\end{array}\right| \neq 0, \quad \delta<\frac{1}{2 \pi} .
$$

The average (in time) value $|J|<1$. The LCE spectrum is “+", “-”, i.e., there is intermixing.

It might seem that we are dealing with an ordinary chaotic strange attractor, but it is not so. A distinctive feature of the considered case is that, despite the contraction, the motion of a representative point of the map (8) is ergodic! As $n \rightarrow \infty$, the point visits any element of the unit square! The evidence of this fact is that the metric dimension of the attractor (the capacity according to Kolmogorov) equals 2. Although the density of points of the attractor is not uniform in the unit square but it is nowhere equal to 0 . Therefore, inspite of the contraction, the attractor of the system (8) is the whole unit square. In this sense Arnold's attractor is not strange as its geometry is not fractal.

Let us consider how the attractor is formed in order to understand in more detail the peculiarities of its structure. Let us choose a small element of the area as a region of initial conditions $\left(0 \leq x_{n} \leq 0.2\right.$, $0 \leq y_{n} \leq 0.2$ ) and observe the evolution of this element while iterating map (8).

Figure 13 represents the sequential images of the initial small square that display the following. Due to the contraction along one direction and the extension along another the initial square evolves into a finite set of "bands" which tend to cover the entire surface of the unit square when iterating. As $n \rightarrow \infty$ we have "the black square".

But as seen from the phase diagram of the attractor shown in Fig. 14, although the points cover the square practically entirely its distribution density is explicitly inhomogeneous! As a quantitative measure of such an inhomogeneity we use the 



FIGURE 13 Evolution of an initial square element in Arnold's map (8) in 1, 3 and 5 iterations, respectively.

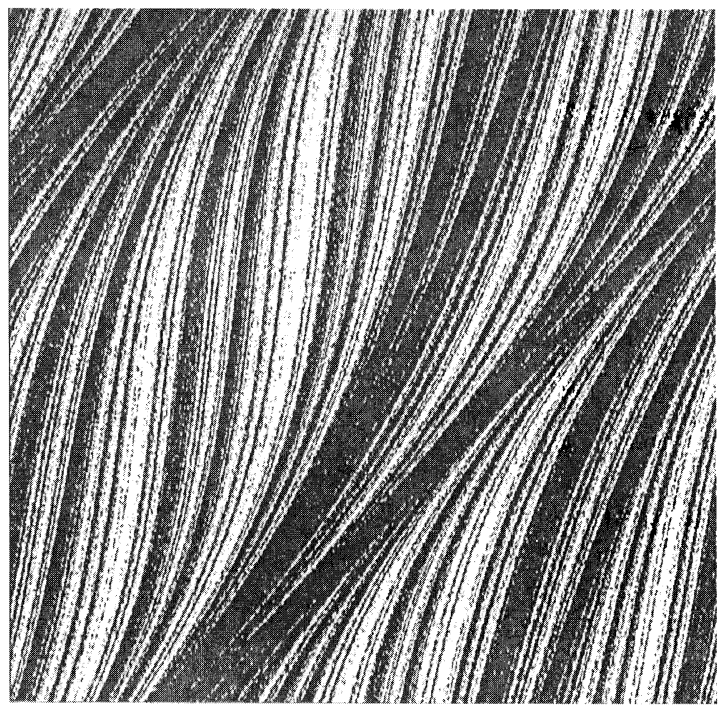

FIGURE 14 Phase diagram of the chaotic nonstrange attractor in the Arnold map (8) for $\delta=0.15$.

information dimension $1 \leq D_{\mathrm{I}} \leq 2$. For instance, for $\delta=0.05, D_{\mathrm{I}} \simeq 1.96$, for $\delta=0.10, D_{\mathrm{I}} \simeq 1.84$. In addition, as we have said, the capacity $D_{\mathrm{C}}=2.0$ (this is a rigorous result of Y. Sinai). As a consequence of inhomogeneity of the probability distribution density of the points on the attractor, the values of an probability-metric dimensions of the Arnold's attractor will lie in the interval $1 \leq D \leq 2$. These dimensions take into account not only geometric but also dynamical properties of the attractor.

CNAs were revealed in a number of other maps on a torus. One can assume that ergodic chaotic motions are typical for diffeomorphisms on a torus. A proof of the existence of CNA in such maps gives the possibility to state that there are flow (differential) system in $\mathfrak{R}^{N}, N \geq 4$ which have the regimes of CNA. However, by the present time CNAs have not been discovered in differential dynamical systems. In this connection, in particular, by now a problem of the possibility of the existence of the chaotic attractor on a threedimensional torus surface embedded into phase space with the dimensionality $N \geq 4$ is still open.

Let us pay attention to the following important fact. As seen from Fig. 14, the chaotic set of the points of map (8) cover densely the unit square of the surface with some continuous probability measure. Therefore, the attractor is the entire unit square! Then how is this fact adjusted with the definition of the attractor as the isolated limit set? What is the region of attraction in this case? Such questions arise because map (8) describes only the attractor and does not contain any information about transient processes. Here we should discuss as follows. Let us choose some differential system in $\mathfrak{R}^{N}(N \geq 4)$ that has a three-dimensional torus $T^{3}$ as its attractor (the system is dissipative!). Consider the structure of phase trajectories on $T^{3}$. In order to do that let us introduce the Poincare section on it. In this case we have a map on a two-dimensional torus $T^{2}$. System (8) is such a map. It models the properties of the limit trajectories lying in the original system on $T^{3}$, i.e., on the attractor. Therefore, Fig. 14 illustrates the structure of the attractor and the region of its attraction is outside of the limits of possibilities described by the discrete model. 


\subsection{Strange Nonchaotic Attractors}

As we have said, strange chaotic attractors possess geometric "strangeness" and intermixing. In other words, complex dynamics of an intermixing system is the reason for the geometric complexity of the corresponding attractor. Nevertheless, in the case of CNA we had to divide these properties, since intermixing cannot always lead to geometrical "strangeness" of the attractor. In this part we will consider the possibility of realizing the opposite situation when the system demonstrates complicated non-periodical oscillatory regime that is asymptotically stable (without intermixing) but the attractor is not regular from the viewpoint of its geometric structure.

One can easily think of examples of nonrobust SNAs. Any strange chaotic attractor at the critical point of its transition to chaos is an example of SNA. Indeed, let us explore, for instance, the Feigenbaum attractor in the well-known logistic map:

$$
x_{n+1}=r x_{n}\left(1-x_{n}\right) .
$$

At the critical point $r^{*}=3.569945$ there appears a limit set of points that has the fractal dimension $D_{\mathrm{C}} \approx 0.548 \ldots$ (a so-called Feigenbaum attractor). In addition, the Lyapunov exponent is equal to zero (there is no chaos!). According to the definition, such an attractor is strange nonchaotic. But it is nonrobust. From the physical point of view, it is interesting to study robust attractors which exist on the set of parameter values of nonzero measure and hold their structure under perturbations. As it has become clear, robust SNAs exist both in differential and discrete dynamical systems (Grebogi et al., 1984; Kapitaniak and Wojewoda, 1993; Anishchenko et al., 1996).

SNAs are typical for dynamical systems driven by quasiperiodic force. Here it is useful to elucidate what the attractor of a non-autonomous system means in our understanding. Assume that an autonomous dynamical system in $\mathfrak{R}^{N}$ is driven by a periodic force with period $T_{0}=2 \pi / \omega_{0}$. We will analyze the Poincare section in a period of the external force. In a secant surface $t=n T_{0}$ each time (for any $n$ ) we will observe some set of points. In this case the attractor is a projection of the set of points in the secant planes, which was obtained for a sequence $n \rightarrow \infty$, on the initial secant surface for $n=0$. It is also possible to use the following method. The original non-autonomous system can be reduced to an autonomous system by introducing new variables and limiting the regions of new variable values by the period duration. Thus, the phase space is expanded and one can use the attractor definition introduced in Section 2.

A feature of the systems with quasiperiodic force is that the introduction of two new independent variables means that we take into consideration two time scales which are not related with the state variables of the original autonomous system and are independent of each other.

In the simplest case, a map where SNA is realized can be written in the form

$$
x_{n+1}=f\left(x_{n}, \phi_{n}, r\right), \quad \phi_{n+1}=\omega+\phi_{n}, \quad \bmod 1,
$$

where $x_{n}$ is a dynamical variable, $\phi_{n}$ is a phase of external force, $r$ is a system parameter (or parameters), $f$ is a nonlinear function that is periodic with respect to $\phi_{n}$ with the period $1, \omega$ is an irrational number. If $\omega$ is irrational, then the forcing will be quasiperiodic because there is no period $k$ such that $f\left(\phi_{n+k}\right)=f\left(\phi_{n}\right)$. Thus, the maps in the form (11) model the dynamics of differential systems with quasiperiodic (two-frequency) external force. There are two characteristic time scales, namely, $k_{1}=1$ (a map iteration) and $k_{2}=1 / \omega, \omega$ is a phase shift during one iteration. Therefore, $\omega$ is called the rotation number that is characterized by the ratio of two frequencies of the quasiperiodic force.

SNA was first revealed and studied in the following map (Grebogi et al., 1984):

$x_{n+1}=\lambda \operatorname{th}\left(x_{n}\right) \cos 2 \pi \phi_{n}, \quad \phi_{n+1}=\omega+\phi_{n}, \quad \bmod 1$. 
An irrational value of the parameter $\omega$ is more often chosen to be equal to the so-called "golden mean": $\omega=0.5(\sqrt{5}-1)$. For the values $\lambda>1$ in the map (12) the existence of SNA was rigorously proved. Besides system (12), SNAs were revealed when quasiperiodic excitation had been applied to the circle map, logistic map, Henon map, etc. The examples of SNA in map (12) and in Feigenbaum map (Heagy and Hammel, 1994)

$$
\begin{aligned}
& x_{n+1}=\alpha\left(1-s \cos 2 \pi \phi_{n}\right) x_{n}\left(1-x_{n}\right), \\
& \phi_{n+1}=\phi_{n}+\omega, \quad \bmod 1
\end{aligned}
$$

are presented in Fig. 15.

The main features of SNA which allow us to extract these objects as a separate class are as follows:

1. Geometric characteristics of SNA. The attractor (for example, on the phase plane) is formed by a curve of an infinite length that is nondifferentiable on the dense set of points. This curve like Peano's curve covers densely a part of the phase plane so that the metric dimension (the capacity) of SNA is strongly equal to 2. But unlike the map (8), in this case one cannot consider that a part of the plane is the attractor since the total measure of points belonging to the attractor is equal to 0 . The fact that information dimension $D_{\mathrm{I}}$ equals 1 (the latter corresponding to the line but not to the plane) indicates this circumstance. Since there is no positive exponent in the LCE spectrum, the Lyapunov dimension of SNA equals 1 . Despite the integer metric dimension, SNA demonstrates as a rule a self-similarity of the structure and, as a consequence, the properties of scaling. All the properties indicated above allow us to speak about the "strange" geometry of SNA.

2. The LCE spectrum of the strange nonchaotic attractor. The system dynamics in the SNA regime is not chaotic as there is no intermixing. In average there is no exponential instability of trajectories on the attractor. The LCE spectrum does not contain a positive exponent. The LCE spectrum signature of phase trajectories on SNA does not differ from the corresponding one of a quasiperiodic motion. However, SNA cannot be considered as a quasiperiodic attractor because, in particular, the local (calculated on an finite time interval) largest LCE spectrum exponent of a trajectory on SNA will be positive. Particularly, it has been proved that the probability that the largest local Lyapunov exponent will be positive is not equal to zero.

3. Spectrum and autocorrelation function. As there is no intermixing in the regime of SNA, the power spectrum does not contain in a rigorous sense the continuous component. At the same time, the spectrum of a trajectory on SNA is not discrete! The spectrum of SNA that is intermediate between discrete and continuous cases has a specific name: a singular continuous spectrum. A feature of the singular continuous spectrum is that it includes a dense set of $\delta$-peaks of the self-similar structure and has the properties of fractals.

Since the spectrum of SNA is not continuous, the autocorrelation function $\Psi(\tau)$ does not tend to zero as $\tau \rightarrow \infty$. For the trajectories on SNA, $\Psi(\tau)$ decreases to some limit nonzero level. Moreover, $\Psi(\tau)$ will demonstrate the scale-invariant properties in the same way as spectrum.

As an example, Fig. 16 represents the spectrum of SNA in system (12) calculated for the coordinate $x(n)$ of the attractor shown in Fig. 15(a) (Pikovsky and Feudel, 1995). As seen from the graph, the spectrum is really an everywhere-dense set of $\delta$-peaks and does not contain a pronounced continuous component. Looking at the shape of the spectrum function $\left|S_{N}\right|$ it is difficult to get sure that the distribution of spectrum components obeys the scale-invariant properties. For this purpose let us consider the autocorrelation function $\Psi(\tau)$ for the attractor in Fig. 15(a) which is shown in Fig. 17 (Pikovsky and Feudel, 1994).

If we compare the dependencies $\Psi(\tau)$ the time intervals $-1000 \leq \tau \leq 1000$ and $1584 \leq \tau \leq 3584$, then we can conclude about the complete 

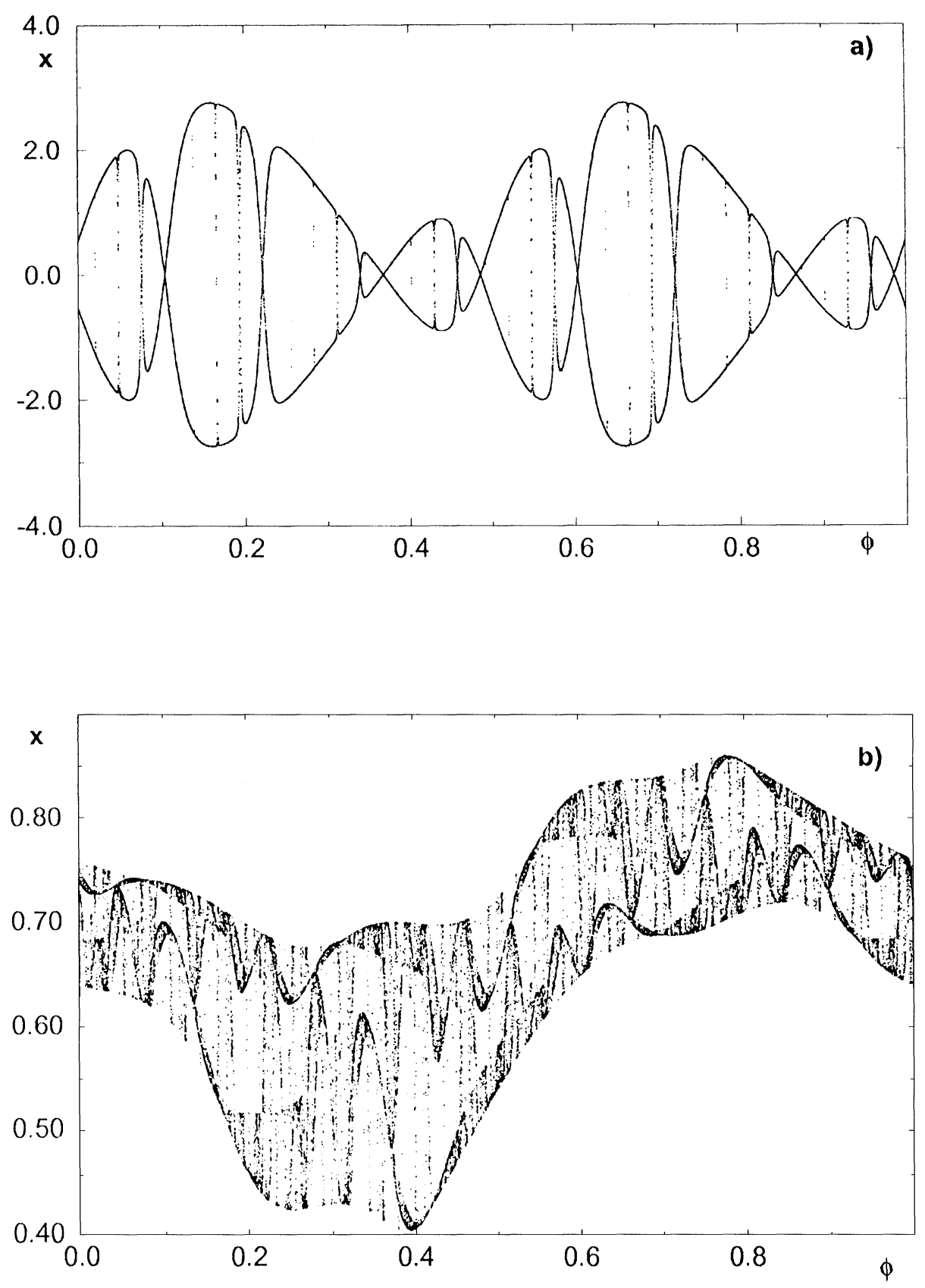

FIGURE 15 Phase diagrams of strange nonchaotic attractors in the map (12) for $\lambda=1.5$ (a) and in the map (13) for $s=0.1$, $\alpha=3.277, \omega=0.5(\sqrt{5}-1)$ (b). 


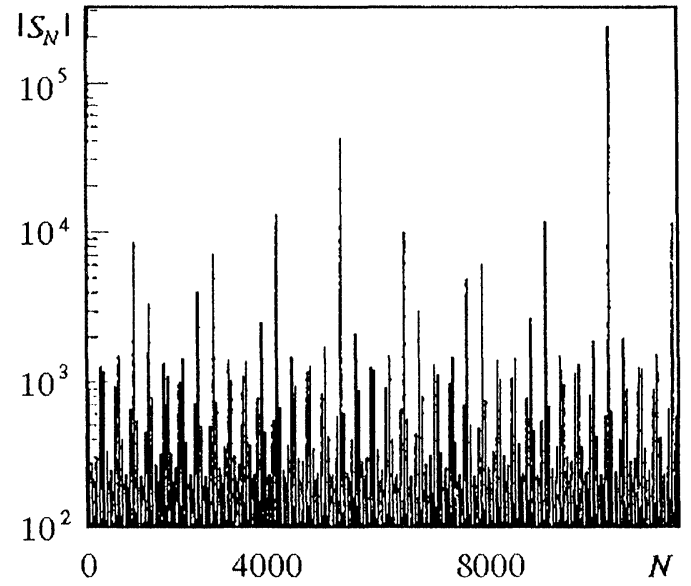

FIGURE 16 Singular-continuous spectrum of SNA in the $\operatorname{map}(12)$ for $\lambda=1.5$.
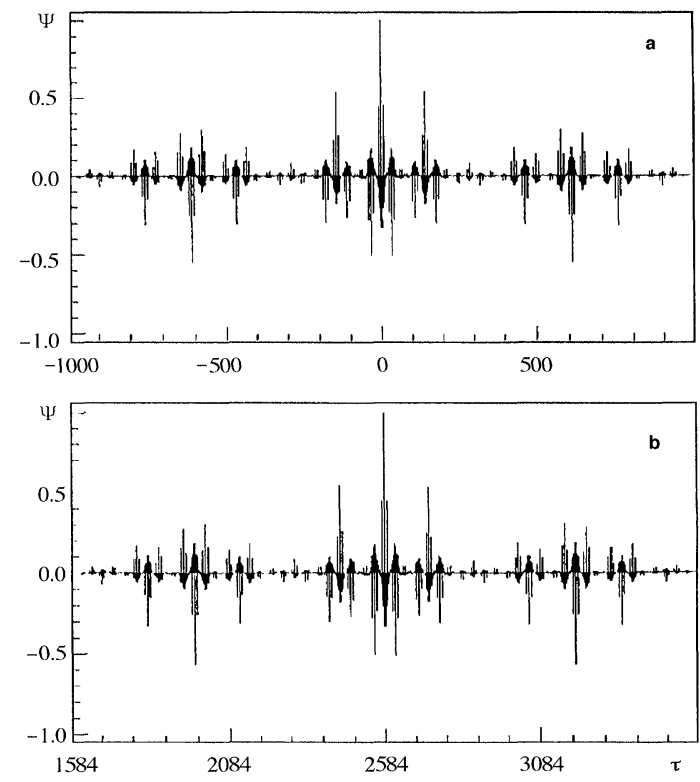

FIGURE 17 Self-similarity of the autocorrelation function of SNA in the system (12).

correspondence of the autocorrelation function structure. The plot for $\Psi(\tau)$ presented in Fig. 17(a) is fully reproduced in Fig. 17(b). This is a consequence of the property of scale invariance. The envelop $|\Psi(\tau)|$ in the SNA regime is a decreasing function that tends to some nonzero limit as $\tau \rightarrow \infty$.

It is important to note that the diagnostics of the SNA regime in numeric simulations is a very difficult and nonstandard task and needs precise calculations to be carried out using a good modern equipment. Otherwise, it is impossible to distinguish the SNA regime and a quasiperiodic regime with a large number of combinative frequencies in the spectrum.

\section{CONCLUSIONS}

The analysis of the structure and properties of attractors of nonlinear dissipative systems as the images of nonperiodic self-oscillations presented in this paper allows us to make the following conclusions:

1. Robust hyperbolic systems and Lorenz type systems demonstrate classical properties of deterministic chaos as nonperiodic exponentially unstable solutions of the corresponding dynamical systems. Strange (or practically strange) attractors are their mathematical images. Their distinctive feature is the fractality of their geometrical structure, the fractal metric dimension and the presence of at least one positive exponent in the LCE spectrum that is a consequence of the intermixing. Robust hyperbolic attractors and Lorenz type attractors are less sensitive to the influence of noise. The basins or attraction of such attractors are smooth and homogeneous. The attractor's properties are not sensitive to the variation of initial conditions.

2. Quasiattractors which include a finite or infinite set of regular and chaotic attracting subsets coexisting for the fixed values of system parameters are more complicated objects. Variation of system parameters can lead to bifurcations of these subsets, whose number may be infinite while there is a finite variation of parameters. The basins of attraction of the co-existing attractors have a fractal geometry. As a result, quasiattractors demonstrate high sensitivity to the changes in initial conditions and the influence of noise. 
3. Exponential instability of individual trajectories and "strange" geometry of an attractor cannot be connected uniquely. There exist the regimes of chaotic (unstable) self-oscillations to which regular, in geometrical sense, attractors correspond. These are the so-called chaotic nonstrange attractors. On the other hand, it is possible to observe nonperiodic stable, according to Lyapunov, oscillations whose corresponding attractor is a strange geometrical object. Here, we deal with the strange nonchaotic attractors.

The ideas and concepts presented in this paper cannot be considered as absolutely noncontradictory and generally accepted. A number of problems described here is up to the present moment a subject of detailed studies and scientific discussions, the latter proving a fundamental significance of the subject under investigation.

\section{Acknowledgements}

The authors express their sincere acknowledgements to Prof. V.N. Belykh, Prof. V. Afraimovich and Dr. T. Vadivasova for the numerous fruitful discussions on a number of mathematical problems. We are also grateful to our colleagues Dr. I. Khovanov and Dr. N. Janson for their help in carrying out some experiments and preparing the manuscript for publication.

This work was partially supported by the grant of the Russian State Committee of High Education N 95-0-8.3-66 and by the common research project of DFG and RFBR N 436 RUS 113/334.

\section{References}

Afraimovich, V. and Shil'nikov, L. (1983). Strange attractors and quasiattractors. In Nonlinear Dynamics and Turbulence (G.I. Barenblatt, G. Iooss and D.D. Joseph, Eds.). Pitman, Boston, London, Melbourne, pp. 1-34.

Afraimovich, V. (1984). Strange attractors and quasiattractors. In Nonlinear and Turbulent Processes in Physics. NY: Gordon and Breach, Harwood Acad. Publ., Vol. 3, pp. $1133-1138$.

Afraimovich, V. (1989). Attractors. In Nonlinear Waves - 1 (A.V. Gaponov, M.I. Rabinovich and J. Engelbrechet, Eds.). Springer-Verlag, Berlin, Heidelberg, pp. 6-28.
Afraimovich, V. (1990). Qualitative theory of stochastic selfoscillations. D.Sc. Thesis. Saratov State University, Saratov, Russia.

Andronov, A., Vitt, A. and Haikin, S. (1981). The Theory of Oscillations. Nauka, Moscow.

Anishchenko, V. (1990). Complex Oscillations in Simple Systems. Nauka, Moscow.

Anishchenko, V. (1995). Dynamical Chaos - Models and Experiments. World Scientific, Singapore.

Anishchenko, V., Vadivasova, T. and Sosnovtseva, O. (1996a). Mechanisms of ergodic torus destruction and appearance of strange nonchaotic attractors. Physical Review E 53(5), $4451-4457$.

Anishchenko, V., Vadivasova, T. and Sosnovtseva, O. (1996b). Strange nonchaotic attractor in autonomous and periodically driven systems. Physical Review E 54(4). 3231-3235.

Arnold, V., Afraimovich, V., Il'yashenko, Yu. and Shil'nikov, L. (1986). The theory of bifurcations. In Modern Problems of Mathematics. Fundamental Directions (V.I. Arnold, Ed.). VINITI, Moscow, Vol. 5, pp. 5-218 (in Russian).

Belykh, V. (1982). Models of discrete systems of phase locking. In Phase Locking Systems (L.N. Belyustina and V.V. Shakhgil'dyan, Eds.). Radio i Svyaz, Moscow, pp. 161-176 (in Russian).

Belykh, V. (1995). Chaotic and strange attractors of twodimensional map. Math. Sbornik 186(3) (in Russian).

Bykov, V. and Shil'nikov, L. (1989). On the boundaries of the domain of existence of the Lorenz attractor. In Methods of Qualitative Theory and Theory of Bifurcations. Gorky State University, Gorky, pp. 151-159 (in Russian).

Cook, A. and Roberts, P. (1970). The Rikitake two-disc dynamo system. In Proc. of Cambridge Philosophical Society, 68, pp. 547-569.

Farmer, J., Ott, E. and Yorke, J. (1983). The dimension of chaotic attractors. Physica D 7, 153.

Gavrilov, N. and Shil'nikov, L. (1972, 1973). About threedimensional dynamical systems close to nonrobust homoclinic curve. Math. Sbornik 88(130), N 8, 475-492; Math. Sbornik 90(132), N 1, 139-156.

Grebogi, C., Ott, E., Pelican, S. and Yorke, J. (1984). Strange attractors that are not chaotic. Physica $D$ 13, 261.

Grebogi, C., Ott, E. and Yorke, J. (1985). Attractors on an N-torus: Quasiperiodicity versus Chaos. Physica D 15, 354-373.

Heagy, J. and Hammel, S. (1994). The birth of strange nonchaotic attractors. Physica D 70, 140-153.

Kapitaniak, T. and Wojewoda, J. (1993). Attractors of Quasiperiodically Forced Systems. World Scientific, Singapore.

Kaplan, J. and Yorke, J. (1979). Chaotic Behavior of MultiDimensional Difference Equations. Lect. Notes in Math. 730, pp. 204-227.

Kifer, Yu. (1974). Some theorems on small random perturbations of dynamical systems. Uspekhi Math. Nauk 29(3), 205 (in Russian).

Lichtenberg, A. and Lieberman, M. (1983). Regular and Stochastic Motion. Springer-Verlag.

Lorenz, E. (1963). Deterministic Nonperiodic Flow. Journal of Atmospheric Sciences 20, 130-141.

Lozi, R. (1978). Un Attracteur Etrange du Type Attracteur de Henon. Journal de Physique 39(C5), 9-10.

Neimark, Yu. and Landa, P. (1989). Stochastic and Chaotic Oscillations. Nauka, Moscow. 
Pikovsky, A. and Feudel, U. (1994). Correlations and spectra of strange nonchaotic attractors. J. Phys. A 27, 5209.

Pikovsky, A. and Feudel, U. (1995). Characterizing strange nonchaotic attractors. CHAOS $\mathbf{5}, 253$.

Plykin, R. (1980). About hyperbolic attractors of diffeomorphisms. Uspekhi Math. Nauk 35(3), 94-104 (in Russian).

Rabinovich, M. and Trubetskov, D. (1984). The Introduction to the Theory of Oscillations and Waves. Nauka, Moscow.

Ruelle, D. and Takens, F. (1971). On the Nature of Turbulence. Commun. Math. Phys. 20, 167-192.

Shil'nikov, L. (1980). The theory of bifurcations and the Lorenz model. In The Hopf Bifurcation and Its Applications (J. Marsden and M. McCracken, Eds.). Mir, Moscow, pp. 317-335 (in Russian).
Shil'nikov, L. (1993). Strange attractors and dynamical models. Journal of Circuits, Systems, and Computers 3(1), 1-10.

Schuster, H. (1984). Deterministic Chaos. Physik-Verlag GmbH, Weinheim (F.R.G.).

Smale, S. (1967). Differential dynamical systems. Bull. Am. Math. Soc. 73, 747-817.

Strelkova, G. and Anishchenko, V. (1997). Structure and properties of quasihyperbolic attractors. In Proc. of Int. Conf. of COC'97 (St. Petersburg, Russia, August 27-29, 1997), Vol. 2, 345-346.

Williams, R. (1977). The Structure of Lorenz Attractors. Lect. Notes in Math. 615, pp. 94-112. 


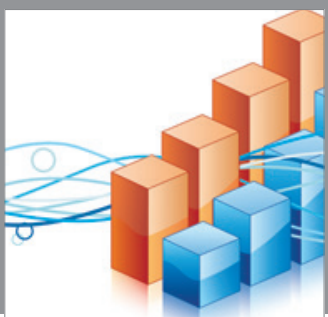

Advances in

Operations Research

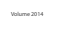

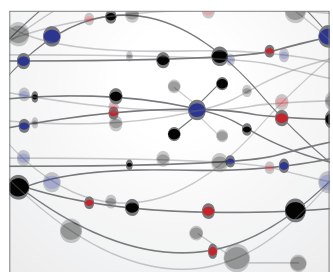

\section{The Scientific} World Journal
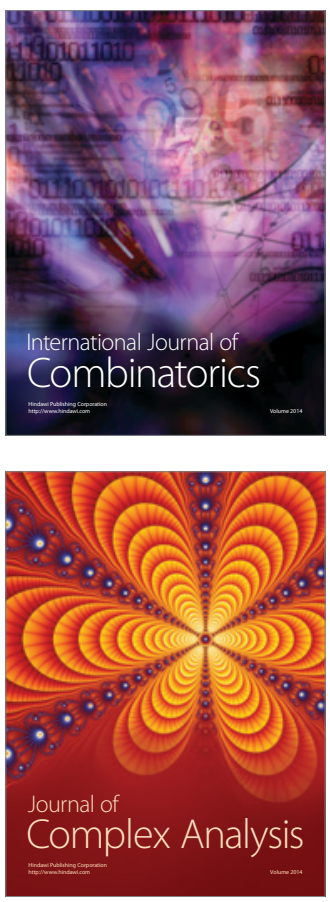

International Journal of

Mathematics and

Mathematical

Sciences
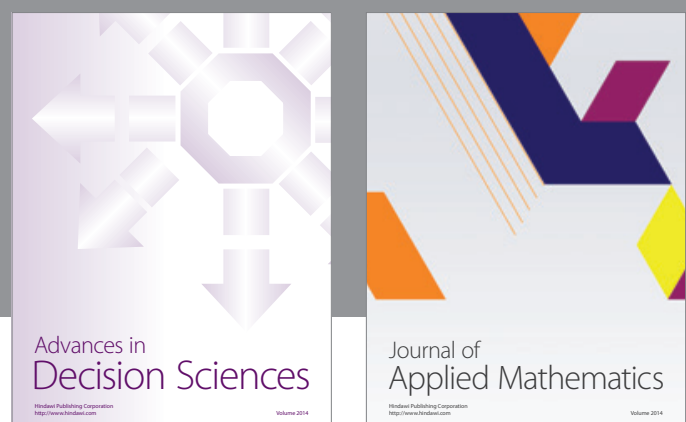

Journal of

Applied Mathematics
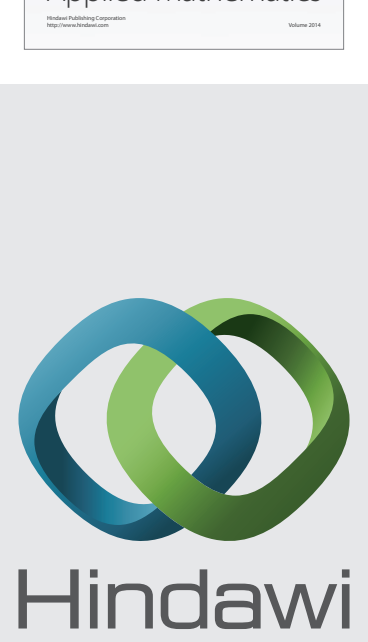

Submit your manuscripts at http://www.hindawi.com
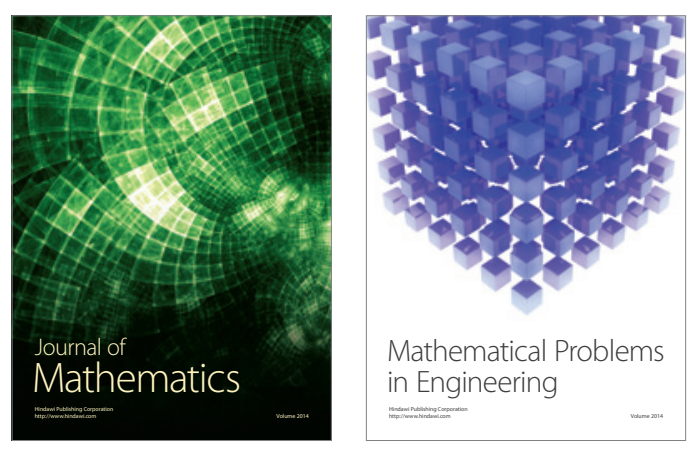

Mathematical Problems in Engineering
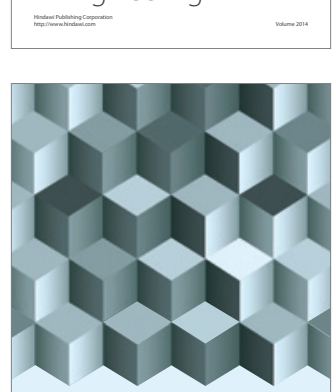

Journal of

Function Spaces
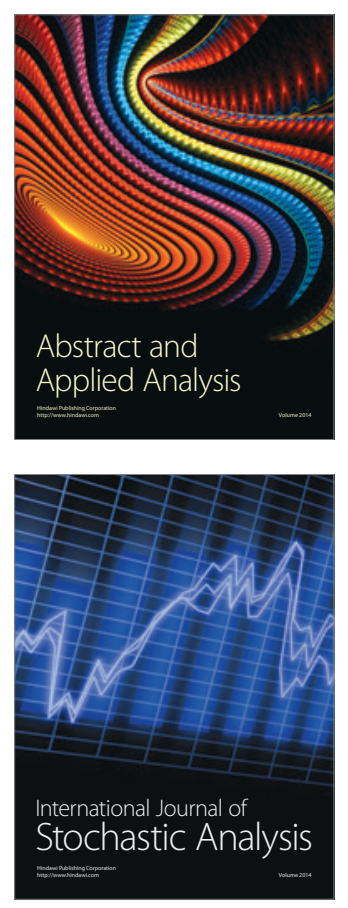

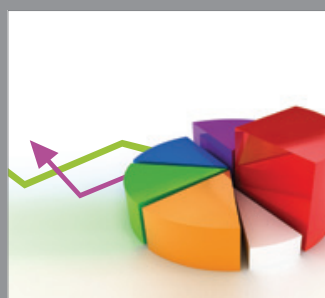

ournal of

Probability and Statistics

Promensencen
